\title{
Party drug to mainstream medicine; the transition of ketamine
}

\author{
N Ravindran, A Ravindran
}

\begin{abstract}
A significant proportion of patients with major depressive disorder are refractory to approved interventions. The first report of significant and rapid benefit of intravenous (IV) ketamine for treatment resistant depression (TRD) appeared in 2000. Since then, there have been multiple reports on the efficacy and tolerability of ketamine formulations for TRD, in particular for single-dose IV administration, which is supported by meta-analyses. The benefit for oral and other non-IV forms remains preliminary. There is preliminary evidence that ketamine has anti-suicidal properties. The adverse effects of ketamine are often mild, brief, and not clinically significant, and include dissociation, sedation, and changes in blood pressure
\end{abstract}

and heart rate. Recently, the S-enantiomer esketamine has been developed, evaluated, and approved for use in North America as adjunct to antidepressants for TRD. Intranasal esketamine has ease of use but the IV form has the advantage of cost. Both are equally effective and tolerable with few drug interactions, and are indicated for adult patients with TRD. Informed consent, medical assessment, supervised setting and monitoring by trained personnel are mandatory. The increased risk of misuse, diversion, and addiction has been recognized with the need for education, responsible practice, and ongoing research.

SL J Psychiatry 2020; 11(1): 1-4
Ketamine reached its prominence as a safe anesthetic agent in the mid 1960's but transitioned into a party drug when its dissociative and hallucinogenic properties were discovered by the club culture (1). By 1980, ketamine use was strongly discouraged purportedly because of its psychotomimetic properties (2). The transition of ketamine to a magical antidepressant began when Berman and colleagues first reported its marked and rapid benefit for treatment-resistant depression (TRD) (3). Ketamine is now hailed as the most important advance in the treatment of depression since the discovery of tricyclics.

Major depressive disorder (MDD) is now recognized as a significant public health issue and a leading cause of disability worldwide (4). In spite of available first line interventions that include antidepressants and psychotherapy, at least a third of patients fail to achieve remission (5). Furthermore, antidepressants need significant lag time to benefit, while evidence-based psychotherapies are often difficult to access and costly (5). This is especially true in low- and middle-income countries (LMICs). Thus, there is an unmet need for cost-effective novel interventions with superior efficacy and rapid relief of symptoms. In light of accumulating evidence ketamine appears to be one such agent with promise (6-8).

\section{How does ketamine produce its antidepressant effects?}

While ketamine's antidepressant mechanisms are not fully elucidated, its predominant effect is on the glutaminergic system, where it acts as an antagonist of N-methyl-D aspartate (NMDA) receptors (9). Additionally, its effect on multiple other neurotransmitter systems, including dopaminergic, gabaminergic, opioid and serotonergic systems have been recognized (10). It has been hypothesized that neuroprotection related to increased neurotrophic factor and protein synthesis, as well as sustained synaptogenesis in depression related neural circuits, may underlie the antidepressant benefit of ketamine (10). The enhanced synaptic plasticity and connectivity is suggested to produce a synergistic benefit with psychological treatments to improve clinical impact (10).

\section{How can ketamine be administered? Is it effective in alleviating symptoms of TRD?}

An anesthetic agent with proven safety, ketamine is commonly administered through the intravenous (IV) route, it can also be administered intramuscularly (IM), sublingually (SL), orally and intranasally (IN). The bioavailability of non-IV ketamine varies widely with the 
route of administration, with oral forms having the lowest levels, followed by SL, IN and IM thus making dose comparisons difficult $(11,12)$. Unfortunately, there are few reports on the relationship between route of administration, bioavailability and plasma levels.

The intravenous route of administration is the most studied for the acute treatment of TRD, with the majority of data coming from single infusions that produced rapid antidepressant effects within hours and lasting up to a week. Such benefit has been confirmed by several metaanalyses of a significant number of RCTs $(6-8,13)$. The dose of ketamine used ranged from 0.5 to $1 \mathrm{mg}$ per $\mathrm{kg}$, usually infused over 30-45 minutes. Most studies reported the higher dose (1mg per $\mathrm{kg}$ ) to be safe and tolerable, with greater likelihood of a more sustained response and a lower relapse risk. Investigations of the benefit of repeated infusions may produce better response and more sustained benefit, but is less studied. Three small RCTs found early evidence for the benefit of adjunctive oral ketamine in TRD. However, high heterogeneity and variability in the degree of benefit (probably related to differences in bioavailability) limit interpretation (13). The IN formulation of racemic ketamine holds promise with better bioavailability versus the oral form but requires a specific delivery system for its use (14). Interestingly, several small investigations have reported favourably on the use of IN racemic ketamine for maintenance after initial treatment with IV for TRD (15). Overall, the evidence base for the benefit of non-IV forms of racemic ketamine remains limited. Efficacy wise, the initial finding of Berman et al. has since been well replicated in randomized controlled trials of IV ketamine in TRD subjects, and further confirmed beyond doubt by several meta-analyses $(3,16,6-8,13)$.

Racemic ketamine exists as two enantiomers, S and R, with the former being four times more potent at inhibiting NMDA receptors (9). While there is a racemic $S$ form, Janssen has developed esketamine IN version with the trade name Spravato ${ }^{\circledR}$. Several large RCTs and a metaanalysis confirm its superiority over placebo in the acute treatment of TRD when used as an adjunct to first line antidepressants, and is currently approved for use in the USA and Canada, though its relative high cost may discourage use in LMICs $(7,13)$.

\section{What adverse effects should be anticipated when using ketamine?}

The common and clinically relevant adverse effects experienced with subanesthetic doses of IV ketamine include psychotomimetic, cardiovascular, and neurological, among others (7). For most patients, such effects are mild and short-lasting, resolving within an hour of infusion, and severe dissociative symptoms are rare (17). Dissociative symptoms are often described as dream like or as dizziness or "confusion", and often accompanied by mild perceptual disturbances.
Cardiovascular effects of increased blood pressure and heart rate are similarly mild and transient and tend to occur after 30 minutes of the start of infusion, with less than $10 \%$ of patients reporting significant increase (18). In general, ketamine does not have significant effect on CYP450 isoenzymes and is unlikely to cause clinically significant drug-drug interactions (19). Concomitant use of first line antidepressant agents is considered safe, although data related to monoamine oxidase inhibitors are not yet available. However, ketamine may increase the effect of sedatives, opioids, anesthetics and alcohol (20).

Potential long-term risks of ketamine have been recognized, almost exclusively in chronic abusers (21). Although relatively uncommon, chronic abuse has been reported to cause cognitive dysfunction and cystitis with multiple urinary symptoms, referred to as "ketamine bladder" (21). These adverse effects have not been reported with therapeutic use of ketamine.

\section{What criteria are helpful for selecting patients for ketamine treatment?}

Until now, most off-label and experimental use has targeted patients with TRD who have had trials of multiple medications and/or electroconvulsive therapy. Expert consensus recommends that ketamine be considered primarily for adult patients with TRD, commonly defined as failed trials of two or more antidepressants from different classes, with at least one trial of adjunctive augmentation, as per standard protocol (4).

There is early evidence that ketamine may have specific anti-suicidal effects (22). Reductions in suicidal ideation have been reported with single as well as multiple infusions, and confirmed in a recent meta-analysis (6). The anti-suicidal effects appear to be independent of the antidepressant benefit, but need further systematic evaluation (22).

Evidence for the benefit and safety of ketamine for depression in paediatric and geriatric age groups is limited and needs further study (8). Similarly, safety of ketamine use during pregnancy and breastfeeding has not been established. Ketamine should be avoided in patients with symptoms or history of psychoses, as well as in those with cardiovascular disease and other unstable medical conditions (8).

\section{What clinical protocol is appropriate for ketamine use?}

Prior to treatment, as part of good clinical practice, patients should undergo full physical examination and appropriate clinical investigations. It is imperative that patients be well informed of its risks and benefits. For safety reasons, IV and IN ketamine are best administered in a supervised setting where rescue medications for 
medical emergencies and, facilities for cardiac monitoring are available. As well, most centres strongly recommend the immediate or quick availability of advanced cardiac life support (ACLS), and the presence of a physician trained and experienced in airway management.

The usual duration of patient stay for IV and IN treatments is estimated to be 2-3 hours, during which the subject should be observed closely. During treatment, blood pressure, heart rate, pulse oximetry, and level of consciousness should be checked and documented prior to, during (every 30 minutes), and post-infusion for at least one hour. The physiological measures should be checked and mental state assessed prior to discharge. Patients should be cautioned against driving for the next 24 hours (23).

Though it is recognized that ketamine should be administered in a supervised medical setting, such standards have not been well established. Ketamine administration has been largely administered through research protocols in academic centers by trained personnel with available ACLS, thus there is a need to establish protocols for real-world settings, that strike a pragmatic balance between patient safety, access and cost of treatment.

It is well recognized that there is significant recreational use and chronic abuse and dependence in vulnerable groups and certain regions of the world. Thus, the risk of diversion and addiction cannot be ruled out with noninjectable formulations of ketamine. It has been strongly recommended that patient and family education should be integral to any ketamine treatment program. Furthermore, medical facilities should have available secure storage to discourage misuse.

\section{What innovations and cautions are being foreseen with ketamine use?}

While its benefit is clear, it is well recognized that there are potential risks with the growing use of ketamine. Among them is the dramatic increase in its off label use as well as high patient demand triggered by media reports, both of which have ethical and legal considerations. The precedent for successful off label use of psychotropics and the high prevalence of TRD has led to increased patient demand. Frequent off-label use of ketamine may compromise safety and increase abuse potential, thus there is a need to balance benefit and potential harm (24). Any commercial or public investment in large multicenter RCTs with racemic ketamine is unlikely. Data to improve clinical practice is likely to be derived from smaller well conducted studies and naturalistic data from open use (25). The increasing prevalence of suicide, particularly in youth, provides high incentive to further evaluate the anti-suicidal benefit of ketamine. Similarly, its potential benefit for depression spectrum conditions including reactive distress and other "contextual indications" need study (26). A recent publication by Singh et al. provides an excellent overview of clinical use, practice pointers, and recommendations for its regulation and monitoring (24). A second publication by Krystal et al. (25) provides an excellent research perspective and vision for future opportunities. The literature in general cautions against overly restrictive practices to realize ketamine's full potential. They further emphasize the need for extensive education and responsible practices.

\section{Acknowledgements}

The assistance of Dr. Angela Paric and Ms. Sonali Amerasekera is gratefully acknowledged.

\section{Conflicts of interest}

Both authors received a research grant from Janssen to conduct a trial of esketamine for treat resistant depression. Neither received personal remuneration from Janssen.

$\mathrm{N}$ Ravindran is a co-author and A Ravindran is a senior co-author on the Canadian Network for Mood and Anxiety Treatments (CANMAT) guidelines for ketamine use.

Nisha Ravindran, Arun Ravindran, Campbell Family Mental Health Research Centre, Centre for Addiction and Mental Health \& Department of Psychiatry, University of Toronto, 250 College Street, Toronto. ON. M5T1R8, Canada

Corresponding Author: A Ravindran

Email: arun.ravindran@camh.ca

http://orcid.org/0000-0002-1655-2753

\section{References}

1. Corssen G, Domino EF. Dissociative anesthesia: further pharmacologic studies and first clinical experience with the phencyclidine derivative CI-581. Anesth Analg 1966; 45: $29-40$

2. Hang LC, Rajagopalan S, Mathew SJ. The history of ketamine use and its clinical indications. In: Mathew SJ, Zarate CA, Editors. Ketamine for Treatment-Resistant Depression: The First Decade of Progress. Cham: Springer International Publishing; 2016: 1-12.

3. Berman RM, Cappiello A, Anand A, Oren DA, Heninger GR, Charney DS, et al. Antidepressant effects of ketamine in depressed patients. Biol Psychiatry 2000; 47: 351-4.

4. Lam RW, Kennedy SH, Parikh S V, MacQueen GM, Milev RV, Ravindran AV. Canadian Network for Mood and Anxiety Treatments (CANMAT) 2016 clinical guidelines 
for the management of adults with major depressive disorder: Introduction and methods. Can J Psychiatry 2016; 61: 510-23.

5. Rush AJ, Trivedi MH, Wisniewski SR, Nierenberg AA, Stewart JW, Warden D, et al. Acute and longer-term outcomes in depressed outpatients requiring one or several treatment steps: A STAR*D report. Am J Psychiatry 2006; 163: 1905-17.

6. Wilkinson ST, Ballard ED, Bloch MH, Mathew SJ, Murrough JW, Feder A, et al. The effect of a single dose of intravenous ketamine on suicidal ideation: A systematic review and individual participant data meta-analysis. Am J Psychiatry 2018; 175(2): 150-8.

7. McGirr A, Berlim MT, Bond DJ, Fleck MP, Yatham LN, Lam RW. A systematic review and meta-analysis of randomized, double-blind, placebo-controlled trials of ketamine in the rapid treatment of major depressive episodes. Psychol Med 2015; 45: 693-704.

8. Kishimoto T, Chawla JM, Hagi K, Zarate CA, Kane JM, Bauer M, et al. Single-dose infusion ketamine and nonketamine $\mathrm{N}$-methyl-d-aspartate receptor antagonists for unipolar and bipolar depression: A meta-analysis of efficacy, safety and time trajectories. Psychol Med 2016; 46: 1459-72.

9. McGirr A, LeDue J, Chan AW, Xie Y, Murphy TH. Cortical functional hyperconnectivity in a mouse model of depression and selective network effects of ketamine. Brain 2017; 140: 2210-25.

10. Abdallah CG, Adams TG, Kelmendi B, Esterlis I, Sanacora G, Krystal JH. Ketamine's mechanism of action: a path to rapid-acting antidepressants. Depress Anxiety 2016; 33: 689-97.

11. Clements JA, Nimmo WS, Grant IS. Bioavailability, pharmacokinetics, and analgesic activity of ketamine in humans. J Pharm Sci 1982; 71: 539-42.

12. Malinovsky JM, Servin F, Cozian A, Lepage JY, Pinaud M. Ketamine and norketamine plasma concentrations after i.v., nasal and rectal administration in children. $\mathrm{Br} \mathrm{J}$ Anaesth 1996; 77: 203-7.

13. Rosenblat JD, Carvalho AF, Li M, Lee Y, Subramanieapillai M, McIntyre RS. Oral ketamine for depression: A systematic review. J Clin Psychiatry 2019 16; 80(3): $18 \mathrm{r} 12475$.

14. Lapidus KAB, Levitch CF, Perez AM, Brallier JW, Parides MK, Soleimani L, et al. A randomized controlled trial of intranasal ketamine in major depressive disorder. Biol Psychiatry 2014; 76: 970-6.
15. Lee V, Archer S, Chrenek C, Swainson J. A response to: Repeated intranasal ketamine for treatment resistant depression: The way to go? Results from a pilot randomised controlled trial. J Psychopharmacol 2019; 33: 258-9.

16. Phillips JL, Norris S, Talbot J, Birmingham M, Hatchard T, Ortiz A, et al. Single, repeated, and maintenance ketamine infusions for treatment-resistant depression: A randomized controlled trial. Am J Psychiatry 2019; 176: 401-9.

17. Acevedo-Diaz EE, Cavanaugh GW, Greenstein D, Kraus C, Kadriu B, Zarate CA, et al. Comprehensive assessment of side effects associated with a single dose of ketamine in treatment-resistant depression. J Affect Disord 2020; 263: $568-75$.

18. Riva-Posse P, Reiff CM, Edwards JA, Job GP, Galendez GC, Garlow SJ, et al. Blood pressure safety of subanesthetic ketamine for depression: A report on 684 infusions. J Affect Disord 2018; 236: 291-7.

19. Andrade C. Ketamine for depression, 5: Potential pharmacokinetic and pharmacodynamic drug interactions. J Clin Psychiatry 2017; 78: e858-61.

20. Sandoz. Mementine product monogrpah. Boucherville, QC: Sandoz; 2015.

21. Sassano-Higgins S, Baron D, Juarez G, Esmaili N, Gold M. A review of ketamine abuse and diversion. Depress Anxiety 2016; 33(8): 718-27.

22. Williams NR, Heifets BD, Bentzley BS, Blasey C, Sudheimer KD, Hawkins J, et al. Attenuation of antidepressant and antisuicidal effects of ketamine by opioid receptor antagonism. Mol Psychiatry 2019; 24: 1779-86.

23. Hayley AC, Green M, Downey LA, Stough CKK, Keane $\mathrm{M}$, Shiferaw B, et al. The acute and residual effects of escalating, analgesic-range doses of ketamine on driving performance: A simulator study. Prog Neuro-Psychopharmacology Biol Psychiatry 2018; 86: 83-8.

24. Singh I, Morgan C, Curran V, Nutt D, Schlag A, McShane R. Ketamine treatment for depression: opportunities for clinical innovation and ethical foresight. Lancet Psychiatry 2017; 4: 419-26.

25. Krystal JH, Abdallah CG, Sanacora G, Charney DS, Duman RS. Ketamine: A Paradigm Shift for Depression Research and Treatment. Neuron 2019; 101: 774-8.

26. Andrade C. Ketamine for depression, 2: Diagnostic and contextual indications. J Clin Psychiatry 2017; 78: e555-8. 